\title{
Interhemispheric connectivity revealed by diffusion tensor imaging fiber tracking derived from navigated transcranial magnetic stimulation maps as a sign of language function at risk in patients with brain tumors
}

\author{
*Nico Sollmann, MD, 1,2 Chiara Negwer, MD, ${ }^{1,2}$ Lorena Tussis, BSc, ${ }^{1,2}$ Theresa Hauck, ${ }^{1,2}$ \\ Sebastian Ille, MD, ${ }^{1,2}$ Stefanie Maurer, ${ }^{1,2}$ Katrin Giglhuber, ${ }^{1,2}$ Jan S. Bauer, MD, ${ }^{3}$ Florian Ringel, MD, \\ Bernhard Meyer, MD, ${ }^{1}$ and Sandro M. Krieg, MD, MBA ${ }^{1,2}$ \\ ${ }^{1}$ Department of Neurosurgery, ${ }^{2}$ TUM-Neuroimaging Center, and ${ }^{3}$ Section of Neuroradiology, Department of Radiology, Klinikum
rechts der Isar, Technische Universität München, Germany
}

\begin{abstract}
OBJECTIVE Resection of brain tumors in language-eloquent areas entails the risk of postoperative aphasia. It has been demonstrated via navigated transcranial magnetic stimulation (nTMS) that language function can partially shift to the unaffected hemisphere due to tumor-induced plasticity. Therefore, this study was designed to evaluate whether interhemispheric connectivity (IC) detected by nTMS-based diffusion tensor imaging-fiber tracking (DTI-FT) can be used to predict surgery-related aphasia in patients with brain tumors.
\end{abstract}

METHODS Thirty-eight patients with left-sided perisylvian brain lesions underwent cortical language mapping of both hemispheres by nTMS prior to awake surgery. Then, nTMS-based DTI-FT was conducted with a fractional anisotropy (FA) of 0.01 and 0.2 to visualize nTMS-based IC. Receiver operating characteristics were calculated for the prediction of a postoperative (irrespective of the preoperative state) and a new surgery-related aphasia by the presence of detectable IC.

RESULTS Language mapping by nTMS was possible in all patients. Seventeen patients (44.7\%) suffered from surgeryrelated worsening of language performance (transient aphasia according to 3-month follow-up in 16 subjects [42.1\%]; new permanent aphasia according to 3-month follow-up in 1 patient [2.6\%]). Regarding the correlation of aphasia to nTMS-based IC, statistically significant differences were revealed for both evaluated FA values. However, better results were observed for tractography with an FA of 0.2 , which led to a specificity of $93 \%$ (postoperative aphasia) and $90 \%$ (surgery-related aphasia). For postoperative aphasia, the corresponding OR was $0.1282(95 \% \mathrm{Cl} 0.0143-1.1520)$, and for surgery-related aphasia the OR was $0.1184(95 \% \mathrm{Cl} 0.0208-0.6754)$.

CONCLUSIONS According to these results, IC detected by preoperative nTMS-based DTI-FT might be regarded as a risk factor for surgery-related aphasia, with a specificity of up to $93 \%$. However, because the majority of enrolled patients suffered from transient aphasia postoperatively, it has to be evaluated whether this approach distinctly leads to similar results among patients with permanent language deficits. Despite this restriction, this approach might contribute to individualized patient consultation prior to tumor resection in clinical practice.

http://thejns.org/doi/abs/10.3171/2016.1.JNS152053

KEY WORDS aphasia; brain tumor; diffusion tensor imaging; fiber tracking; interhemispheric connectivity; transcranial magnetic stimulation; oncology

\footnotetext{
ABBREVIATIONS $C C=$ corpus callosum; $D C S=$ direct cortical stimulation; $D T I=$ diffusion tensor imaging; $E H I=$ Edinburgh Handedness Inventory; FA = fractional anisotropy; fMRI = functional MRI; FN = false-negative; FP = false-positive; FT = fiber tracking; IC = interhemispheric connectivity; NPV = negative predictive value; $\mathrm{nTMS}$ = navigated transcranial magnetic stimulation; 01 = first object; $\mathrm{O} 2$ = second object; PPV = positive predictive value; $\mathrm{PTI}=$ picture-to-trigger interval; RMT = resting motor threshold; $\mathrm{ROC}=$ receiver operating characteristic; $\mathrm{ROI}=$ region of interest; $\mathrm{TN}=$ true-negative; $\mathrm{TP}=$ true-positive.

SUBMITTED August 31, 2015. ACCEPTED January 14, 2016.
}

INCLUDE WHEN CITING Published online April 1, 2016; DOI: 10.3171/2016.1.JNS152053.

* Drs. Sollmann and Negwer contributed equally to this work. 
$\mathrm{T}$ HE resection of brain tumors that are located within so-called eloquent areas entails the inherent risk of causing a functional deficit that reduces the patient's quality of life. Regarding tumors that are located within language-eloquent brain regions, the risk of surgery-related aphasia is one of the main factors that can limit the overall extent of resection significantly and therefore has to be considered during patient counseling. To achieve a reasonable balance between resection and preservation of neurological function, the application of preand intraoperative neuroimaging and mapping methods is essential..$^{7,9,10,46}$ Regarding mapping of human language, navigated transcranial magnetic stimulation (nTMS) has already proven to be a helpful and reliable tool that correlates well with direct cortical stimulation (DCS), the gold standard in terms of functional mapping. ${ }^{21,36,37,56}$ Furthermore, the use of nTMS mapping data for diffusion tensor imaging-fiber tracking (DTI-FT) principally allows for the detection of subcortical language-related pathways. ${ }^{50}$ In this context, nTMS-based DTI-FT represents a combination of functional and structural neuroimaging that is able to provide individualized information on cortical and subcortical functional anatomy.

These data can be used during surgery by implementation in the neuronavigation; they can also be used to evaluate patients by visualizing their individual functional anatomy in the vicinity of the tumor. Yet, these data could also be of value to allow for the assessment of patients' risk for surgery-related aphasia in surgical decision making. In this context, various studies have already investigated language reorganization within the human brain due to intracranial pathologies, and language plasticity was reported to occur as a partial shift of language function to the unaffected hemisphere. ${ }^{3,6,20,42,60}$ Such a compensatory shift between hemispheres has to be mediated somehow, and it seems obvious that the corpus callosum (CC), which represents interhemispheric connectivity (IC) as the largest white matter structure that connects homologous and nonhomologous brain regions, ${ }^{13,14,58}$ might play a crucial role in this context. Indeed, there are initial studies using functional MRI (fMRI) available that report on enhanced IC both in the context of motor and language compensation mechanisms, ${ }^{31,39}$ which underlines the presumable involvement and modulation of IC in the context of plastic brain reshaping due to intracranial pathologies in general.

Additionally, there is evidence specifically derived from recent nTMS trials showing that language function can partially shift to the unaffected hemisphere during the course of tumor-induced plasticity, ${ }^{20,42}$ indicating that the healthy hemisphere seems to be able to partially take over language function from its impaired homolog, most likely emerging from the attempt to compensate for a potential loss of function. Thus, IC detected by nTMS could be an essential factor that might be altered in the course of such an interhemispheric shift. If so, preoperative assessment of nTMS-based IC would allow for functional evaluation and surgical risk stratification in the individual patient. Thus, the present study was designed to evaluate whether IC detected by nTMS-based DTI-FT correlates with surgery-related aphasia in patients undergoing left-sided perisylvian brain tumor resection during awake surgery.

\section{Methods \\ Ethics Considerations}

The present study was approved by the local institutional review board and was conducted in accordance with the Declaration of Helsinki. Written informed consent was obtained from all patients.

\section{Patient Selection}

Overall, 38 patients were enrolled in the present investigation between 2011 and 2014. Patients were eligible for participation if they were over 18 years old and were diagnosed with a tumor localized within perisylvian areas of the left hemisphere (i.e., classic language-eloquent regions). Exclusion criteria were general nTMS exclusion criteria (e.g., the presence of a cochlear implant or cardiac pacemaker). In addition, patients with severe aphasia that did not allow for nTMS language mapping were not included.

\section{Study Design}

All patients underwent MRI, preoperative repetitive nTMS for cortical language mapping of the left and right hemispheres, and nTMS-based DTI-FT. Tumor resection was performed during awake surgery including intraoperative neuronavigation, intraoperative DCS, and subcortical language mapping. ${ }^{21,35,36,47,55}$ Aphasia was assessed during the preoperative, postoperative (on the 5th postoperative day), and follow-up examinations (3 months after surgery) using a modified grading scale as published earlier $(0=$ no aphasia, $1=$ mild aphasia, $2=$ moderate aphasia, 3 = severe aphasia, A = nonfluent aphasia, and $\mathrm{B}=$ fluent aphasia). ${ }^{21,52}$ Postoperative aphasia was defined as any grade of language impairment at the 5th postoperative day, regardless of preoperative language function. Surgery-related aphasia was defined as any change in language performance when comparing pre- and postoperative language function (Table 1). All aphasia grading was done by a medical doctor with neurosurgical training.

\section{Imaging Parameters}

Scanning was conducted with a 3-T MR scanner (Achieva 3T, Philips Medical Systems) through the use of an 8-channel phased-array head coil. Imaging included a T2-weighted FLAIR (TR/TE 12,000/140 msec, voxel size $0.9 \times 0.9 \times 4 \mathrm{~mm}^{3}$, acquisition time $3 \mathrm{~min}$ ) and a $3 \mathrm{D}$ T1-weighted gradient echo sequence (TR/TE 9/4 msec, 1 $\mathrm{mm}^{3}$ isovoxel covering the whole head, acquisition time $6 \mathrm{~min} 58 \mathrm{sec}$ ) with and without intravenous application of gadopentetate dimeglumine (Magnograf, Marotrast $\mathrm{GmbH}$ ) for contrast enhancement. Furthermore, DTI sequences were acquired with 6 (TR/TE $7571 / 55 \mathrm{msec}$, spatial resolution of $2 \times 2 \times 2 \mathrm{~mm}^{3}$, b-values of 0 and 800 , acquisition time $2 \mathrm{~min} 15 \mathrm{sec}$ ) or 15 (TR/TE 10,737/55 msec, spatial resolution of $2 \times 2 \times 2 \mathrm{~mm}^{3}$, b-values of 0 and 800, acquisition time 6 min $26 \mathrm{sec}$ ) orthogonal diffusion directions.

In each subject, motion artifacts of the acquired DTI data were adjusted using the software of the MR scanner. The contrast-enhanced 3D gradient echo sequences and 


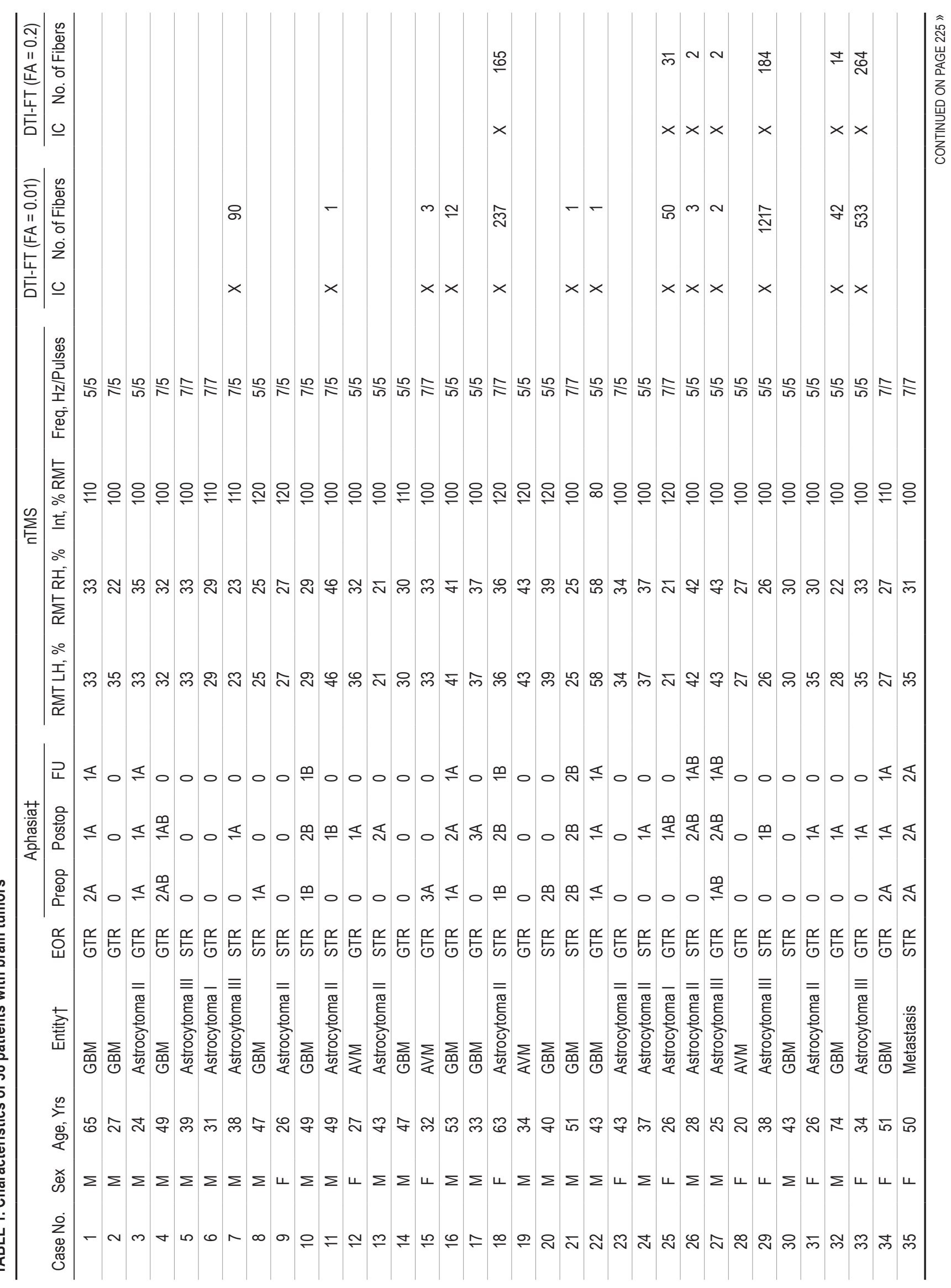




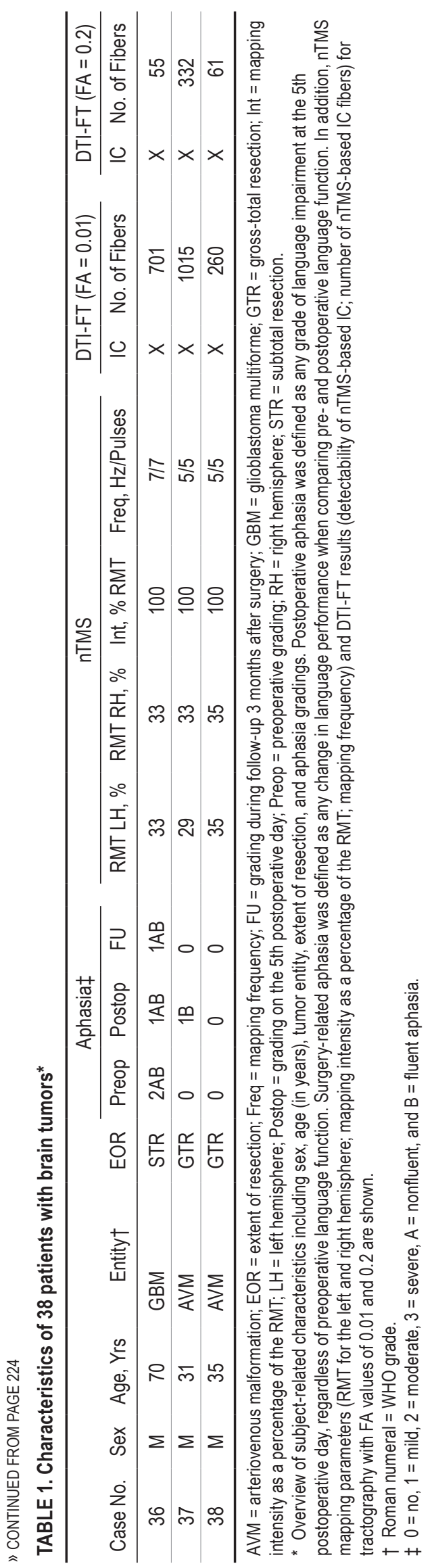

DTI sequences were transferred to the Nexstim eXimia NBS system (version 3.2.2 or version 4.3, Nexstim Oy) and to a Brainlab iPlan Net server (version 3.0.1, Brainlab AG).

\section{Navigated Transcranial Magnetic Stimulation}

Language mapping of both hemispheres was performed with the eXimia NBS system, version 3.2.2 or 4.3, in combination with a NEXSPEECH module (Nexstim Oy). First, a contrast-enhanced 3D gradient echo sequence of the patient was uploaded to the system's software because it was needed for individualized neuronavigation. The head position of each patient was then tracked by reflectors fastened to the head with an elastic strap and an infrared tracking camera (Polaris Spectra). Moreover, the coil position was followed by reflectors fixed to the back side of the magnetic coil. This setup allowed for controlled coil movement and coil detection during nTMS. ${ }^{24,44}$ In this context, the current approach for nTMS language-mapping trials has been repeatedly described. ${ }^{15,22,23,30,41,42,54,56}$

Briefly, the resting motor threshold (RMT) was first determined to be able to individually adjust the stimulation intensity during language mapping. Muscle electrodes (Neuroline 720, Ambu) were placed over the abductor pollicis brevis muscle. We identified the most excitable spot in the precentral gyrus to assess the exact RMT value, which is commonly defined as the lowest stimulation intensity that elicits motor evoked potentials greater than 50 $\mu \mathrm{V}$ in amplitude in $50 \%$ of stimulation trials. ${ }^{43}$ We determined the RMT separately for each hemisphere because both hemispheres were examined.

During language mapping, the patients were instructed to name everyday objects as quickly and precisely as possible. ${ }^{22,30,42,54}$ The objects were displayed on a screen and the assortment was similar to the objects of Snodgrass and Vanderwart. ${ }^{49}$ Initially, the subjects had to perform the object-naming task twice without stimulation, and objects that were misnamed or did not elicit clear responses were discarded. ${ }^{22,30,42,54}$ The remaining objects constituted the baseline and were used in the mapping session, and they were presented in randomized order during stimulation. If a patient was not able to sufficiently perform baseline testing due to aphasia or incompliance according to the evaluation of the examiner and at least 1 medical doctor with profound nTMS experience, he or she was excluded from the present study.

Each mapping was performed according to a protocol that has proven to be reliable in recent nTMS investigations. ${ }^{21,22,30,36,51,54,56}$ The nTMS pulses were applied timelocked to the objects (display time $700 \mathrm{msec}$, interpicture interval $2500 \mathrm{msec}$ ), and the picture-to-trigger interval (PTI) was $300 \mathrm{msec}$ in the first 10 patients and $0 \mathrm{msec}$ in the remaining 28 patients. There is evidence for both PTIs, ${ }^{17,41,45,62}$ but we decided to switch to $0 \mathrm{msec}$ with regard to a recent evaluation of the advantages of $0 \mathrm{msec}$ for nTMS language mapping when compared with awake mapping. ${ }^{23}$ Both hemispheres were examined in consecutive order, and each stimulation target was stimulated 3 times before moving the coil to the next stimulation spot during the interpicture interval. A video camera recorded both the baseline testing and the language mapping for later offline analysis. $22,30,42,54$ 
The stimulation videos were systematically searched for naming errors by comparing them to the corresponding baseline recordings as described previously. ${ }^{15,22,30,36,54}$ This was done by at least 1 experienced and trained investigator, and a linguist was available for support in unclear cases. All detected naming errors were classified as no-response errors (complete lack of naming response), performance errors (articulatory or language production errors), neologisms (production of nonexistent words), phonological paraphasias (unintended phonemic modification of the target word), or semantic paraphasias (substitution of a semantically related word for the target word). These error categories are most frequently used during nTMS analyses and have been described precisely in recent publications..$^{22,30,42,54}$ Each stimulation point at which a naming error of 1 of these categories was elicited was defined as a language-positive spot.

After analysis of nTMS mapping data, we transferred all left- and right-sided language-positive spots to an external Brainlab iPlan Net server via the DICOM standard. Hence, all language-positive spots derived from preoperative nTMS mapping were implemented into the neuronavigation system, and these functional landmarks were used for resection planning and intraoperative resection guidance in all patients. Tumor resection was then performed during an awake surgery approach, which included intraoperative neuronavigation, intraoperative DCS, and intraoperative subcortical stimulation, according to current practice. . $1,35,36,47,55^{-1}$

\section{Diffusion Tensor Imaging-Fiber Tracking}

As described above, all language-positive spots (stimulation spots at which naming errors of the described categories were elicited) were transferred to an external Brainlab iPlan Net server to allow for neuronavigation planning and DTI-FT. Only error points were uploaded to the server, whereas language-negative spots (stimulation spots at which no naming errors were elicited during nTMS) were not taken into account. The software performs DTI-FT based on a deterministic tracking approach, which reconstructs subcortical white matter fibers on the basis of the fiber assignment by continuous tracking (FACT) principle..$^{32}$ This software has been used repeatedly for nTMS-based DTI-FT and represents one of the most common and well-distributed clinical tractography tools in neurosurgery. ${ }^{12,19,50}$ First, the left-hemispheric and right-hemispheric language-positive nTMS spots were uploaded as 2 separate data files, and both were fused with the same preoperatively obtained FLAIR, gradient echo, and DTI sequences. Eddy current correction was applied throughout. The stack of merged data was then used for intraoperative neuronavigation, and it constituted the basis for later DTI-FT. Each imported nTMS spot was displayed as a column consisting of 3 solitary points belonging to the same language-positive nTMS spot at 0,5 , and $10 \mathrm{~mm}$ from the cortical surface in the data set.

Next, objects eligible for subsequent DTI-FT had to be determined for the software. Thus, the file containing all left-hemispheric language-positive spots was displayed, and the whole group of these individual spots was defined as the first object (O1). The same was done with the right- hemispheric language-positive spots in an analogous way: the corresponding file was opened, and the entire group of right-hemispheric spots was defined as the second object (O2). Consequently, 2 single objects (O1 and $\mathrm{O} 2)$ were created. Then, 2 separate regions of interest (ROIs) were generated from these objects by simply defining each object as a single ROI. Accordingly, O1, which was previously generated from the left-hemispheric data file, became the left-hemispheric ROI, whereas $\mathrm{O} 2$ became the right-hemispheric ROI (Fig. 1). During the step of ROI definition, an additional rim of $5 \mathrm{~mm}$ was added to each languagepositive spot. As a result, 2 ROIs were visualized: The first ROI solely consisted of left-hemispheric language-positive spots, whereas the second one only included right-hemispheric points derived from the nTMS data. The immediate definition of $\mathrm{O} 1$ and $\mathrm{O} 2$ as ROIs replaces the conventional drawing method for ROI definition.

Subsequently, tracking of white matter fibers that were connecting these 2 ROIs was performed with a minimum fiber length of $40 \mathrm{~mm}$. Regarding the fractional anisotropy (FA), tracking was conducted separately with 2 values (0.01 and 0.2 ) until predefined stop criteria were reached (FA value, fiber angulation $>30^{\circ}$ ). In this context, 0.01 represents the lowest FA value technically possible for DTI-FT, whereas 0.2 led to reliable results in nTMS-based tractography of motor pathways in recent trials..$^{12,19}$ With respect to these adjustments, the DTI-FT algorithm calculated all fibers in between the 2 ROIs. If no fibers were tracked, the condition was defined as IC- (no IC detectable; Fig. 1 left), whereas the visualization of at least 1 fiber connecting the 2 ROIs was defined as IC+ (IC detectable; Fig. 1 right). In cases of IC+, the software's output consisted of a directionally encoded color map of white matter fibers, which all originated from either the left- or right-hemispheric ROI and went to the contralateral ROI. By definition, for later correlation with clinical data, nTMS-based IC was present when there was at least 1 fiber visualized that connected the left-hemispheric ROI with the right-hemispheric ROI (IC+), whereas there was no nTMS-based IC registered when there were no fibers present between these 2 ROIs (IC-). Furthermore, the software displayed the overall number of tracked fibers. If no nTMS-based IC was present (IC-), the corresponding fiber number was 0 .

To additionally evaluate the quality of DTI scans (especially in cases where no nTMS-based IC was revealed), DTI-FT was performed separately with a manually drawn $\mathrm{ROI}$ at the CC without the language-positive nTMS spots to track callosal fibers.

In addition to the language-positive nTMS spots that were displayed during intraoperative neuronavigation, DTI-FT results in the affected hemisphere were also accessible during surgery. However, the results of the nTMSbased IC assessment were not provided before or during tumor resection.

\section{Statistical Analysis}

All statistical data analyses were performed using GraphPad Prism (GraphPad Prism 6.04). Regarding patient and mapping-related characteristics as well as fiber numbers, the mean $\pm \mathrm{SD}$, median, minimum, and maximum values are reported. Differences between left- and 

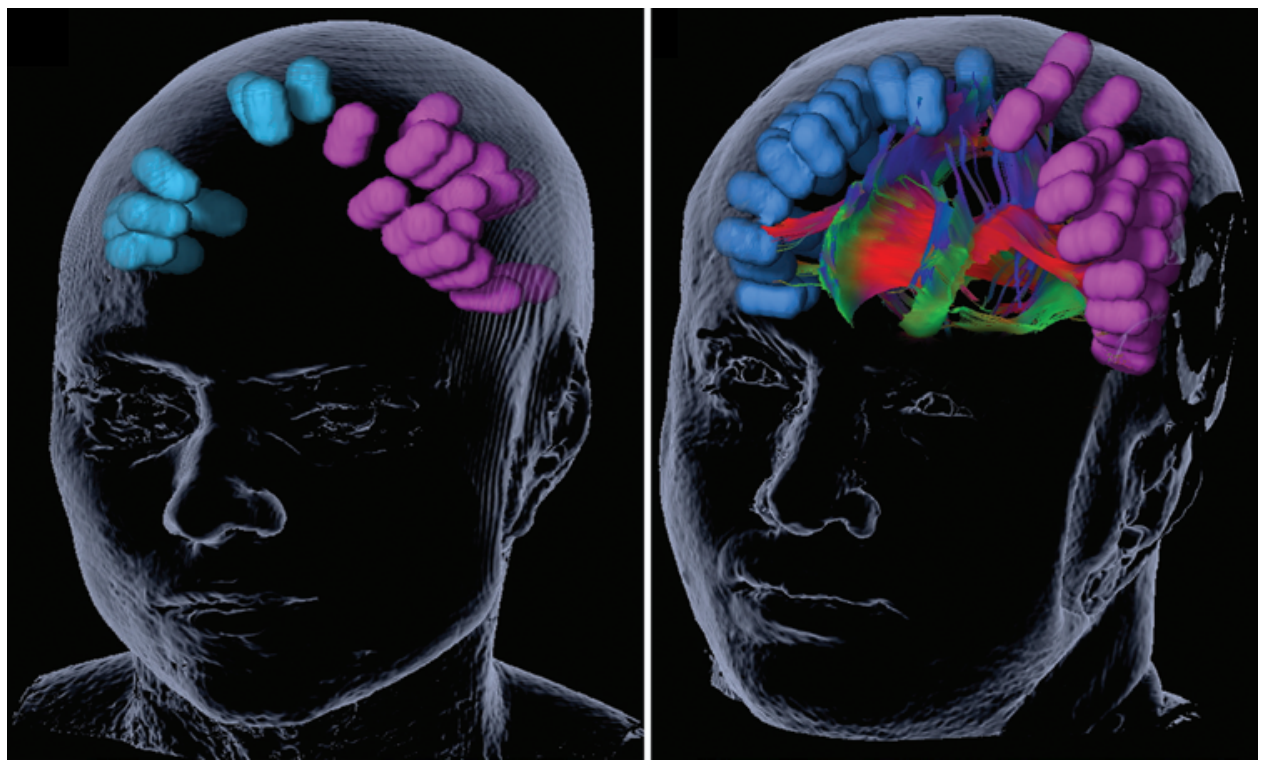

FIG. 1. Illustrative cases of DTI-FT based on nTMS mapping results. The left panel shows unsuccessful DTI-FT for detection of IC (no IC detectable $=\mathrm{IC}-$ ), whereas the right panel shows a case in which DTI-FT led to visualization of IC (IC detected $=I C+$ ). In the right panel, detected IC is visualized by directionally encoded subcortical white matter fibers between the left- and right-hemispheric ROIs. Left-hemispheric language-positive nTMS spots constituted the left-hemispheric ROI (shown in purple), whereas the right-hemispheric ROI consists of all right-hemispheric language-positive nTMS points (depicted in blue). Language-positive nTMS spots are specific cortical points that were prone to a certain kind of naming error during nTMS mapping in combination with an object-naming task. Figure is available in color online only.

right-hemispheric mapping parameters were tested by an independent samples t-test (statistical significance $\mathrm{p}<0.05$ ).

Regarding aphasia, contingency tables were generated based on the total number of patients diagnosed with or without aphasia preoperatively, on the 5th postoperative day, and during follow-up after 3 months in relation to the detectability of IC by nTMS-based DTI-FT. To assess whether nTMS-based IC could serve as a predictive parameter for aphasia in patients with brain tumors, the OR and the corresponding 95\% CI were calculated. Moreover, a chi-square test revealed whether differences in these characteristics were significant or not (statistical significance $p$ $<0.05)$.

In addition, we calculated receiver operating characteristics (sensitivity, specificity, positive predictive value [PPV], and negative predictive value [NPV]) based on the obtained DTI-FT results. ${ }^{26}$ In this context, aphasia represented the ground truth, and the following conditions were defined as follows: 1) true-positive (TP) - IC was detected and aphasia was found; 2) true-negative (TN)-no IC was detected and no aphasia was found; 3) false-positive (FP) - IC was detected and no aphasia was found; and 4) false-negative (FN) - no IC was detected and aphasia was found.

Based on these 4 conditions, the potential influence of the PTI on the obtained tracking results was examined because 2 different PTIs were used during language mapping in the present study $(300 \mathrm{msec}$ and $0 \mathrm{msec})$. This was done by additional contingency analyses systematically comparing these conditions between the patients who were mapped with $300 \mathrm{msec}$ and $0 \mathrm{msec}$. Again, a chi-square test was used to assess statistical significance (statistical significance $\mathrm{p}<0.05$ ).

\section{Results}

\section{Patient Characteristics}

The median age of the cohort, which consisted of 12 women $(31.6 \%)$ and 26 men (68.4\%), was 39 years (range 20-74 years). According to the Edinburgh Handedness Inventory (EHI), 35 subjects (92.1\%) were right-handed (EHI $80.1 \pm 21.5$ ), whereas the remaining 3 patients $(7.9 \%$ ) were left-handed or ambidextrous (EHI $-63.0 \pm 47.8$ ). All enrolled patients were considered suitable for nTMS language mapping.

Seventeen patients $(44.7 \%)$ suffered from surgery-related worsening of language performance. Of these $17 \mathrm{pa}-$ tients, $16(42.1 \%)$ were diagnosed with transient aphasia, meaning that language function resolved at least to the preoperative state during the 3-month follow-up interval. Furthermore, 1 patient $(2.6 \%)$ was diagnosed with a new permanent aphasia that did not resolve to the initial preoperative status during the 3-month follow-up interval. Table 1 gives an overview of subject-related characteristics, including tumor entities and aphasia grades for each patient.

\section{Navigated TMS Mapping}

Left-hemispheric and right-hemispheric cortical language mapping by nTMS was achieved successfully in all patients enrolled. Each patient had at least 1 language-positive point on each hemisphere with respect to the definition of language-positive nTMS spots given in the Methods section, and language-positive spots were found close to the tumor in all patients with surgery-related worsening of language function. Furthermore, left-hemispheric nTMS resulted in language-positive spots in all left-handed or ambidextrous patients, and left-hemispheric intraoperative 
DCS elicited clear naming errors in these cases as well. During nTMS mapping, no adverse events were observed.

Furthermore, the mean left-hemispheric RMT was $33.2 \% \pm 7.4 \%$ (range $21 \%-58 \%$ ) and the analogous righthemispheric RMT was $32.4 \% \pm 7.7 \%$ on average (range $21 \%-58 \% ; \mathrm{p}=0.6712$ ). The mapping intensity related to the individual RMT ranged from $80 \%$ to $120 \%$ RMT according to the mapping protocol, with a mean of $104.0 \% \pm$ $9.0 \%$. Table 1 summarizes nTMS mapping parameters for all individuals separately.

\section{Diffusion Tensor Imaging-Fiber Tracking}

Using an FA value of 0.01, nTMS-based IC was detected in 16 of 38 patients (IC+42.1\%), whereas no connectivity was found in the remaining 22 patients (IC- 57.9\%). During DTI-FT with the CC as the ROI, numerous fibers were detected in all patients. Within the group of patients in whom connectivity was identified, the mean number of visualized interhemispheric fibers was $260.5 \pm 394.7$ (range 1-1217 fibers; Table 1).

Furthermore, tracking with an FA value of 0.2 resulted in detection of nTMS-based IC in 10 of 38 patients ( $\mathrm{IC}+$ $26.3 \%$ ), whereas no connectivity was revealed in 28 patients (IC- 73.7\%). The corresponding mean number of visualized interhemispheric fibers was $111.0 \pm 118.2$ (range 2-332 fibers; Table 1).

Table 2 compares the number of patients suffering from aphasia with the number of patients in whom no aphasia was found in relation to the presence of nTMS-based IC for both FA values used. Regarding the comparison for postoperative and surgery-related aphasia for $\mathrm{FA}=0.01$, there were statistically significant differences (postoperative aphasia: $\mathrm{p}=0.0161$, OR 0.1429, 95\% CI 0.0261-0.7831; surgery-related aphasia: $\mathrm{p}=0.0111$, OR $0.1705,95 \% \mathrm{CI}$ 0.0415-0.7008).

TABLE 2. Interhemispheric connectivity and aphasia*

\begin{tabular}{|c|c|c|c|c|c|c|}
\hline \multirow[b]{2}{*}{ Aphasia } & \multicolumn{3}{|c|}{$F A=0.01$} & \multicolumn{3}{|c|}{$F A=0.2$} \\
\hline & $\mathrm{IC}+$ & IC- & $p$ Value & $\mathrm{IC}+$ & IC- & $p$ Value \\
\hline Preop & & & 0.6456 & & & 0.4752 \\
\hline No & 9 & 14 & & 7 & 16 & \\
\hline Yes & 7 & 8 & & 3 & 12 & \\
\hline Postop & & & 0.0161 & & & 0.0404 \\
\hline No & 2 & 11 & & 1 & 13 & \\
\hline Yes & 14 & 11 & & 9 & 15 & \\
\hline Follow-up & & & 0.1687 & & & 0.5045 \\
\hline No & 9 & 17 & & 6 & 20 & \\
\hline Yes & 7 & 5 & & 4 & 8 & \\
\hline Surgery-related & & & 0.0111 & & & 0.0090 \\
\hline No & 5 & 16 & & 2 & 19 & \\
\hline Yes & 11 & 6 & & 8 & 9 & \\
\hline
\end{tabular}

* Comparison between the number of patients suffering from aphasia and the number of patients in whom no aphasia was found correlated to the presence of IC (IC detected = IC+; no IC detectable $=$ IC-) for both FA values used in the present study. The analysis differentiates between preoperative, postoperative, follow-up, and surgery-related aphasia. There were statistically significant differences for the postoperative $(F A=0.01, p=0.0161 ; F A=0.2, p=0.0404$ ) and surgery-related aphasia $(F A=0.01, p=0.0111 ; F A=0.2, p=0.0090)$.
Concerning the corresponding results of the second FA adjustment used in the present study $(\mathrm{FA}=0.2)$, there were also statistically significant differences found for postoperative and surgery-related aphasia (postoperative aphasia: $\mathrm{p}=0.0404$, OR 0.1282 , 95\% CI 0.0143-1.1520; surgeryrelated aphasia: $\mathrm{p}=0.0090$, OR $0.1184,95 \%$ CI $0.0208-$ 0.6754; Table 2).

Sensitivity, specificity, PPV, and NPV for the 2 FA values to predict postoperative and surgery-related aphasia by preoperative nTMS-based IC are displayed in Table 3 and Fig. 2. The figure shows that all data points are clearly above the line $\mathrm{y}=\mathrm{x}$ (random performance) and are located within the left or upper-left part of the graph, reflecting comparatively high TP rates in combination with rather low FP rates.

Regarding a potential influence of the PTI on tractography results, we compared $\mathrm{TP}, \mathrm{TN}, \mathrm{FP}$, and $\mathrm{FN}$ values between the patients that were mapped with $300 \mathrm{msec}$ and $0 \mathrm{msec}$ for both FA values used in the present study. Based on these 4 conditions, the comparison between $300 \mathrm{msec}$ and $0 \mathrm{msec}$ for $\mathrm{FA}=0.01$ was not significant for postoperative aphasia $(\mathrm{p}=0.1107)$, but it became statistically significant for surgery-related aphasia $(\mathrm{p}=0.0387)$. Concerning tracking with $\mathrm{FA}=0.2$, the comparisons for postoperative and surgery-related aphasia were not statistically significant $(\mathrm{p}=0.1557$ and $\mathrm{p}=0.1072$, respectively).

\section{Discussion}

\section{Navigated TMS-Based DTI-FT Related to Aphasia}

The present study explores whether IC detected by nTMS-based DTI-FT can be used to predict surgery-related aphasia in patients with brain tumors. With regard to language function on the 5th postoperative day of our patient cohort, there were statistically significant differences revealed for both DTI-FT adjustments (Table 2). Furthermore, nTMS-based IC was found to a variable degree but less frequently in patients without postoperative aphasia compared with the group of patients suffering from postoperative aphasia. A similar correlation of nTMS-based IC and aphasia was also found for surgeryrelated language impairment. However, all patients who were diagnosed with postoperative worsening of language function suffered from transient aphasia, except for 1 patient who suffered from permanent aphasia (Table 1). This finding seems to restrict the implications of our findings to transient aphasia.

Because nTMS-based IC was not detected in each enrolled patient, we have to be aware of the fact that structural connection between hemispheres is, of course, present in all patients regardless of their grades of language deficits, which was proven by DTI-FT with the CC as the ROI for DTI scan quality assessment in the present trial. In this context, the present study does not claim that anatomical IC is not present in patients where nTMS-based IC was not detectable. Instead, it shows that no detectable IC exclusively based on DTI-FT using individually assessed language mapping data might allow one to draw conclusions about the individual aphasia risk.

Many studies using different neuroimaging modalities have reported on language reorganization within the hu- 
TABLE 3. Sensitivity, specificity, PPV, and NPV*

\begin{tabular}{|c|c|c|c|c|}
\hline \multirow[b]{2}{*}{ Characteristic } & \multicolumn{2}{|r|}{$F A=0.01$} & \multicolumn{2}{|r|}{$\mathrm{FA}=0.2$} \\
\hline & $\%$ w/ Postop Aphasia & $\%$ w/ Surgery-Related Aphasia & $\%$ w/ Postop Aphasia & $\%$ w/ Surgery-Related Aphasia \\
\hline PPV & 88 & 69 & 90 & 80 \\
\hline NPV & 50 & 73 & 46 & 68 \\
\hline Sensitivity & 56 & 65 & 38 & 47 \\
\hline Specificity & 85 & 76 & 93 & 90 \\
\hline
\end{tabular}

man brain due to intracranial pathologies. ${ }^{3,6,20,42,60}$ In this context, language plasticity was reported to occur as a partial shift of language function to the unaffected brain hemisphere. This principally suggests an interhemispheric reorganization of language function to compensate for impaired cerebral language function. ${ }^{3,6,20,42,60}$ At least since the early split-brain examinations, it has been known that the $\mathrm{CC}$ as the main anatomical correlate of structural IC plays an important role, especially for the integrity of language functions. ${ }^{13,14}$

As an interpretation, the detectability of nTMS-based IC might reflect the manifestation of early compensatory mechanisms for recovery of language function via an interhemispheric functional shift when language function is already at risk prior to surgery, because data for DTIFT are solely based on individual functional data in the present study. Basically, this hypothesis is in good agreement with the aforementioned literature on interhemi-

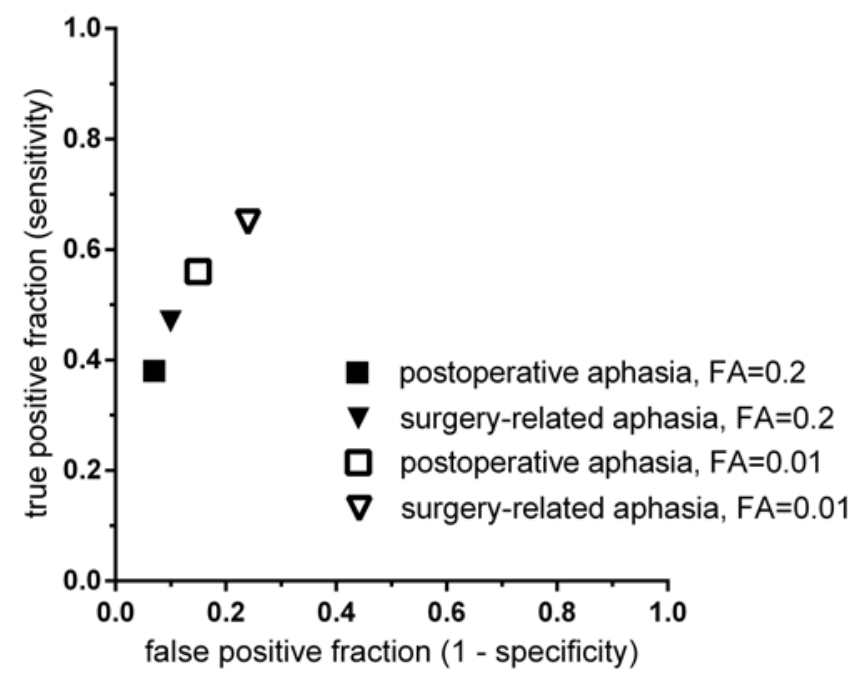

FIG. 2. The TP versus FP fraction for DTI-FT of IC. In this figure, the results for sensitivity are plotted against 1 - specificity for DTI-FT performed with an FA of 0.01 and 0.2 in relation to postoperative and surgery-related aphasia, respectively. For sensitivity, specificity, PPV, and NPV, the following criteria were defined: 1) TP-IC was detected and aphasia was found; 2) TN-no IC was detected and no aphasia was found; 3) FP-IC was detected and no aphasia was found; and 4) FNno IC was detected and aphasia was found. All 4 data points are above $y=x$ (random performance) and are located within the left or upper-left part of the graph, which indicates comparatively high TP rates in combination with low FP rates. spheric language reorganization..$^{3,6,20,42,60}$ Moreover, there are initial fMRI-based studies that report enhanced IC in the context of motor and language compensation mechanisms. ${ }^{31,39}$

Regarding the motor system, it was recently shown that motor recovery after stroke is associated with an enhancement of resting-state IC between motor cortices, suggesting compensatory or reactive neural plasticity. ${ }^{31}$ With respect to the present study, the finding that task-dependent IC of the language network was increased preoperatively within a cohort of patients with epilepsy might be more important. ${ }^{39}$ In this context, a decline during postoperative language tests correlated positively with the strength of preoperative IC, meaning that patients who showed the greatest worsening of language function after surgery were those who previously showed the strongest functional IC. ${ }^{39}$ Hence, stronger preoperative IC appeared to be an unfavorable prognostic biomarker, and one could speculate that a correlate in the form of nTMS-based IC was identified in the present study among patients with brain tumors.

However, it is still a topic of debate how the CC mediates information transfer between hemispheres. ${ }^{58}$ In this context, theories of inhibition and excitation exist, and both seem to be probable candidates to adequately describe callosal function. ${ }^{58}$ With respect to our approach, it could be the case that inhibitory fibers are primarily detected by nTMS-based DTI-FT, which might explain postoperative language worsening due to interhemispheric inhibition. This is speculative and cannot be demonstrated by our present results, but it reflects a hypothetical explanation from a neuroanatomical perspective.

Whereas postoperative aphasia includes both tumor-induced (preoperative) and surgery-related language deficits, surgery-related aphasia solely depicts new or aggravated language impairment due to tumor resection itself. Thus, for these categories, IC detected by nTMS-based DTI-FT could be regarded as a risk factor for the occurrence of at least transient aphasia in patients with brain tumors with a comparatively high specificity, depending on the FA value (Table 3, Fig. 2). However, we are not aware of other literature distinctly investigating IC as a potential aphasia risk factor in patients with brain tumors. Thus, this interpretation has to be confirmed by future studies that include more patients.

We have to note that the reorganization of language function does not seem to be able to compensate for all 
functional impairments in every case. ${ }^{20,42}$ This finding might be reflected by the occurrence of aphasia in some patients in whom nTMS-based IC was not revealed (Table 2). In this context, we should be aware of investigations showing that language shift to the unaffected hemisphere could also lead to a reduction of language abilities in subgroups of patients. ${ }^{48}$ This demonstrates that an ideal correlation or corresponding OR between nTMS-based IC and aphasia might not be achievable per se.

\section{Significance of the Present Study}

In general, the creation of ROIs can be performed according to either anatomical or functional data. However, ROI seeding based on anatomical structures depends on the anatomical knowledge of the examiner and is therefore likely to vary from one operator to another., ${ }^{4,59}$ More importantly, correct identification of anatomical landmarks is challenging in patients suffering from intracranial lesions due to the spatial derangement of subcortical fiber pathways. ${ }^{29,34,40}$ For these reasons, the placement of ROIs based on functional data can be favored, and nTMS mapping is a new modality that can generate data technically suitable for subsequent DTI-FT. In this context, some studies have already successfully used cortical nTMS maps as a source for DTI-FT. ${ }^{5,11,12,19,50,61}$

As described initially, all language-positive spots derived from preoperative nTMS mapping were implemented into the neuronavigation system and were accessible during surgery. Although the present study does not focus on the correlation between preoperative nTMS maps and intraoperative DCS results, it should be mentioned that a good correlation of nTMS and DCS language maps has been demonstrated. ${ }^{16,21,36,53,56}$ Interestingly, the overall agreement between nTMS and DCS language maps was higher when compared with corresponding results of fMRI or magnetoencephalography versus DCS. ${ }^{6,53,56}$ However, nTMS performed with the current stimulation protocols has been shown to be more sensitive and less specific when compared with DCS. ${ }^{16,36}$

Furthermore, there are hints that nTMS language mapping might have a positive impact on clinical parameters because it has been shown to be associated with smaller craniotomies and lower postoperative deficit rates. ${ }^{52}$ The present study explored whether nTMS-based DTI-FT can be used to predict surgery-related aphasia, thereby providing further evidence that nTMS might potentially expand its role as a beneficial tool in neuro-oncological patients.

Overall, the majority of patients with new language deficits were diagnosed with transient aphasia (Table 1). Therefore, we may only be able to state that IC detected by nTMS-based DTI-FT correlates with transient aphasia. This is because only 1 patient suffered from a new permanent aphasia, whereas all other patients showed no new or only transient language impairment. This observation does not imply that our approach is inapplicable to permanent aphasia in general, but rather suggests that larger patient cohorts or multicenter studies are needed to enroll enough patients with permanent deficits to confirm our results. In the present study, the single patient with permanent deficits showed IC, which could be a motivating factor for performing future investigations.
Moreover, although the aphasia grading used in the present study is regarded as sufficiently accurate for most neurosurgical patients, ${ }^{21,52}$ it still seems to be comparatively rough from a more linguistic point of view. Language function is complex, and most postoperative neurosurgical classifications might not cover all aspects of language during the testing period. In other words, some patients might be diagnosed with no impairment even though there is a certain degree of slight or subclinical deficit present. Such impairment is not likely to be relevant for the overall neurosurgical evaluation, but we hypothesize that the use of more sensitive assessments could improve the specificity of our approach. The functional outcome of the patient is important and becomes increasingly crucial for the evaluation of the neurosurgeon's success within the context of the individual onco-functional balance. ${ }^{10}$ Thus, predictive tools such as our approach, in combination with finergrained clinical assessments, seem to be gaining increased importance.

Although IC might primarily be regarded as a risk factor for transient aphasia, it can still be beneficial in daily clinical practice from both the neurosurgeon's and the patient's perspective. From both viewpoints, preoperative risk stratification is valuable because it is intended to inherently support decision making. For the neurosurgeon, detection of nTMS-based IC might create awareness of language function being at risk even in patients for whom mere anatomical imaging may not have suggested it. The result could be more careful and thorough intraoperative stimulation to preserve function. Additionally, preoperative patient consultation might be improved because any statements about the functional outcome could be supported by clinical data derived from individual nTMSbased DTI-FT. Moreover, from the patient's perspective, preoperative knowledge about the presumable postoperative status might influence the decision for or against surgery, in a more enlightened state. The present approach mainly provided data for transient deficits, but prediction of this kind of temporary impairment could strengthen the postoperative acceptance of transient impairments among patients. Although a specificity of up to $93 \%$ was shown in the present study (Table 3 and Fig. 2), further potential benefits of our approach should be carefully tested in upcoming studies.

Although the ROC and corresponding ORs do not reach optimal values, they still allow for functional assessment and consultation of each patient via individualized functional data. In this context, the data points in Fig. 2, which graphically depicts the results of sensitivity against 1 - specificity, can be found above $y=x$ (diagonal line reflecting random performance). Generally, each data point is characterized by a TP-FP rate pair, and high TP rates in combination with low FP rates symbolize perfect classification. ${ }^{26}$ Thus, the upper triangular region above $\mathrm{y}=\mathrm{x}$ contains spots with high TP-low FP values. All of our data points are localized within this area (Fig. 2), indicating comparatively high quality of our testing approach. We are not aware of any previous trials to investigate the surgeryrelated aphasia risk in patients with brain tumors, based on individual functional data. Hence, the present study represents an encouraging first approach for presurgical 
risk stratification in such patients. As aforementioned, this additional information might contribute to individual decision making and the preservation of neurological function as the crucial goal in neurosurgery. ${ }^{10}$

\section{Limitations of DTI-FT}

Although our results are promising and should be valuable for individual assessment of the postoperative aphasia risk, we must be aware of some limitations of the DTI-FT technique. With regard to crossing or kissing white matter fiber bundles, it has been demonstrated that reliable reconstruction of such fiber courses can be challenging (or even impossible) for the tracking algorithms. This is because DTI is still unable to resolve more than a single fiber direction within each imaging voxel., ${ }^{2,8,19,27}$ Especially when the FA value is set comparatively low and, therefore, a high number of fibers is visualized, this limitation can influence the correct fiber visualization. The tracking result might then not necessarily reflect reality. However, new technical approaches, such as q-ball imaging, may compensate for the problem of fiber crossings. ${ }^{33,57}$

Furthermore, DTI-FT of fiber tracts in the vicinity of a brain tumor or within tumor-related edema can be vulnerable to FN results, mainly due to low anisotropy values.,19 In an area where tumor infiltration can be found, the normal white matter architecture of the brain is disordered. More than 1 fiber population probably occupies the same voxel, which makes it complicated to reliably reconstruct white matter pathways. . $^{218,38}$ Yet, our study shows increases in fiber tracts when tumor infiltration is close to functional pathways. Thus, reducing this shortcoming of reduced fiber reconstruction in the vicinity of intracerebral lesions might even increase the PPV and specificity of our presented approach.

Moreover, as already discussed, the majority of patients who showed new language deficits postoperatively were diagnosed with transient aphasia (Table 1). As a consequence, we might only be able to state that IC detected by nTMS-based DTI-FT correlates with transient aphasia, although 1 patient with a permanent deficit and IC was detected. In addition to nTMS-based IC, which might act as a risk factor for surgery-related aphasia in the present study, other parameters may also contribute to surgery-related language deficits. Although the present study enrolled a rather large cohort of patients with brain tumors, it did not systematically account for other factors (e.g., the tumor entity or tumor size) within the scope of a multivariate analysis. To perform this type of statistical evaluation, further studies that enroll more patients are needed.

Regarding a potential influence of the PTI on tractography results, the comparison concerning postoperative aphasia between patients mapped with $300 \mathrm{msec}$ and 0 msec for $\mathrm{FA}=0.01$ and the comparison for postoperative and surgery-related aphasia for FA $=0.2$ were not statistically significant, according to our results. Overall, these analyses suggest that results to predict whether IC detected by nTMS-based DTI-FT correlates with aphasia are not significantly dependent on the PTI used. However, the comparison regarding surgery-related aphasia for FA = 0.01 was statistically significant. Because surgery-related aphasia was comparatively less common among the pa- tients mapped with $300 \mathrm{msec}$ (Table 1), we suppose that this difference was primarily due to unequal aphasia distribution between the patients mapped with $300 \mathrm{msec}$ and 0 msec.

Although these analyses might show that the results presented in this study are not significantly dependent on the 2 PTIs, we believe it is important to state that there is only 1 nTMS PTI comparison study for preoperative mapping available, ${ }^{23}$ and the distinct impact of different PTIs on the intrasubject level has yet to be investigated. As aforementioned, clear evidence for both PTIs is available, ${ }^{17,41,45,62}$ but data on the impact of the PTI on tracking results are lacking. Hence, further studies are probably needed to refine our approach.

\section{Challenges of nTMS-Based DTI-FT}

For detection of language-eloquent cortical and subcortical areas, intraoperative stimulation techniques represent the current gold standard in neurosurgery. Numerous studies have tested the accuracy and reliability of DTI-FT for identification of language pathways in comparison with intraoperative mapping, and a good overall correlation between the modalities has been reported. ${ }^{1,25,28,33}$ However, the question remains whether tractography that is exclusively derived from nTMS language-positive spots accurately reflects reality. Because the present study focused on the prediction of aphasia, this circumstance should not limit the significance of our work. However, further studies are needed to confirm preoperatively gained nTMS-based DTI-FT results by intraoperative subcortical stimulation.

Besides intraoperative validation, further work investigating optimal tracking parameters for nTMS-based DTI-FT is needed. In this context, our study has shown that the number of visualized fibers and specificity as well as sensitivity values clearly depend on the FA value used for tractography, which could be compensated for by the introduction of an optimal tracking protocol for nTMSbased DTI-FT of subcortical fibers associated with language function.

\section{Conclusions}

In general, IC detected by preoperative nTMS-based DTI-FT can be regarded as a risk factor for aphasia in patients with brain tumors with a specificity of up to $93 \%$. However, because the majority of enrolled patients suffered from transient aphasia postoperatively, it has to be evaluated whether our approach leads to similar results among patients with permanent language deficits. Despite this restriction and the limitations of the DTI technique, this novel approach might allow for individual patient consultation prior to tumor resection in clinical practice.

\section{Acknowledgments}

We gratefully acknowledge the support of the Technische Universität München Graduate School's Faculty Graduate Center of Medicine. This study was completely financed by institutional grants from the Department of Neurosurgery and the Section of Neuroradiology.

\section{References}

1. Bello L, Gambini A, Castellano A, Carrabba G, Acerbi F, 
Fava E, et al: Motor and language DTI Fiber Tracking combined with intraoperative subcortical mapping for surgical removal of gliomas. Neuroimage 39:369-382, 2008

2. Berman JI, Berger MS, Chung SW, Nagarajan SS, Henry RG: Accuracy of diffusion tensor magnetic resonance imaging tractography assessed using intraoperative subcortical stimulation mapping and magnetic source imaging. J Neurosurg 107:488-494, 2007

3. Briganti C, Sestieri C, Mattei PA, Esposito R, Galzio RJ, Tartaro A, et al: Reorganization of functional connectivity of the language network in patients with brain gliomas. AJNR Am J Neuroradiol 33:1983-1990, 2012

4. Catani M, Thiebaut de Schotten M: A diffusion tensor imaging tractography atlas for virtual in vivo dissections. Cortex 44:1105-1132, 2008

5. Conti A, Raffa G, Granata F, Rizzo V, Germanò A, Tomasello F: Navigated transcranial magnetic stimulation for "somatotopic" tractography of the corticospinal tract. Neurosurgery 10 (Suppl 4):542-554, 2014

6. Cousin E, Baciu M, Pichat C, Kahane P, Le Bas JF: Functional MRI evidence for language plasticity in adult epileptic patients: Preliminary results. Neuropsychiatr Dis Treat 4:235-246, 2008

7. De Witt Hamer PC, Robles SG, Zwinderman AH, Duffau H, Berger MS: Impact of intraoperative stimulation brain mapping on glioma surgery outcome: a meta-analysis. J Clin Oncol 30:2559-2565, 2012

8. Duffau H: Diffusion tensor imaging is a research and educational tool, but not yet a clinical tool. World Neurosurg 82:e43-e 45, 2014

9. Duffau H, Capelle L, Denvil D, Sichez N, Gatignol P, Taillandier L, et al: Usefulness of intraoperative electrical subcortical mapping during surgery for low-grade gliomas located within eloquent brain regions: functional results in a consecutive series of 103 patients. J Neurosurg 98:764-778, 2003

10. Duffau H, Mandonnet E: The "onco-functional balance" in surgery for diffuse low-grade glioma: integrating the extent of resection with quality of life. Acta Neurochir (Wien) 155:951-957, 2013

11. Forster MT, Hoecker AC, Kang JS, Quick J, Seifert V, Hattingen E, et al: Does navigated transcranial stimulation increase the accuracy of tractography? A prospective clinical trial based on intraoperative motor evoked potential monitoring during deep brain stimulation. Neurosurgery 76:766-776, 2015

12. Frey D, Strack V, Wiener E, Jussen D, Vajkoczy P, Picht T: A new approach for corticospinal tract reconstruction based on navigated transcranial stimulation and standardized fractional anisotropy values. Neuroimage 62:1600-1609, 2012

13. Gazzaniga MS: Forty-five years of split-brain research and still going strong. Nat Rev Neurosci 6:653-659, 2005

14. Gazzaniga MS, Sperry RW: Language after section of the cerebral commissures. Brain 90:131-148, 1967

15. Hernandez-Pavon JC, Mäkelä N, Lehtinen H, Lioumis $P$, Mäkelä JP: Effects of navigated TMS on object and action naming. Front Hum Neurosci 8:660, 2014

16. Ille S, Sollmann N, Hauck T, Maurer S, Tanigawa N, Obermueller $\mathrm{T}$, et al: Combined noninvasive language mapping by navigated transcranial magnetic stimulation and functional MRI and its comparison with direct cortical stimulation. J Neurosurg 123:212-225, 2015

17. Indefrey P: The spatial and temporal signatures of word production components: a critical update. Front Psychol 2:255, 2011

18. Jones DK: Determining and visualizing uncertainty in estimates of fiber orientation from diffusion tensor MRI. Magn Reson Med 49:7-12, 2003

19. Krieg SM, Buchmann NH, Gempt J, Shiban E, Meyer B,
Ringel F: Diffusion tensor imaging fiber tracking using navigated brain stimulation-a feasibility study. Acta Neurochir (Wien) 154:555-563, 2012

20. Krieg SM, Sollmann N, Hauck T, Ille S, Foerschler A, Meyer $\mathrm{B}$, et al: Functional language shift to the right hemisphere in patients with language-eloquent brain tumors. PLoS One 8:e75403, 2013

21. Krieg SM, Sollmann N, Hauck T, Ille S, Meyer B, Ringel F: Repeated mapping of cortical language sites by preoperative navigated transcranial magnetic stimulation compared to repeated intraoperative DCS mapping in awake craniotomy. BMC Neurosci 15:20, 2014

22. Krieg SM, Sollmann N, Tanigawa N, Foerschler A, Meyer B, Ringel F: Cortical distribution of speech and language errors investigated by visual object naming and navigated transcranial magnetic stimulation. Brain Struct Funct [epub ahead of print], 2015

23. Krieg SM, Tarapore PE, Picht T, Tanigawa N, Houde J, Sollmann N, et al: Optimal timing of pulse onset for language mapping with navigated repetitive transcranial magnetic stimulation. Neuroimage 100:219-236, 2014

24. Krings T, Chiappa KH, Foltys H, Reinges MH, Cosgrove GR, Thron A: Introducing navigated transcranial magnetic stimulation as a refined brain mapping methodology. Neurosurg Rev 24:171-179, 2001

25. Kuhnt D, Bauer MH, Becker A, Merhof D, Zolal A, Richter $\mathrm{M}$, et al: Intraoperative visualization of fiber tracking based reconstruction of language pathways in glioma surgery. Neurosurgery 70:911-920, 2012

26. Lasko TA, Bhagwat JG, Zou KH, Ohno-Machado L: The use of receiver operating characteristic curves in biomedical informatics. J Biomed Inform 38:404-415, 2005

27. Le Bihan D, Poupon C, Amadon A, Lethimonnier F: Artifacts and pitfalls in diffusion MRI. J Magn Reson Imaging 24:478-488, 2006

28. Leclercq D, Duffau H, Delmaire C, Capelle L, Gatignol P, Ducros M, et al: Comparison of diffusion tensor imaging tractography of language tracts and intraoperative subcortical stimulations. J Neurosurg 112:503-511, 2010

29. Lehéricy S, Duffau H, Cornu P, Capelle L, Pidoux B, Carpentier A, et al: Correspondence between functional magnetic resonance imaging somatotopy and individual brain anatomy of the central region: comparison with intraoperative stimulation in patients with brain tumors. J Neurosurg 92:589-598, 2000

30. Lioumis P, Zhdanov A, Mäkelä N, Lehtinen H, Wilenius J, Neuvonen T, et al: A novel approach for documenting naming errors induced by navigated transcranial magnetic stimulation. J Neurosci Methods 204:349-354, 2012

31. Liu J, Qin W, Zhang J, Zhang X, Yu C: Enhanced interhemispheric functional connectivity compensates for anatomical connection damages in subcortical stroke. Stroke 46:10451051,2015

32. Mori S, van Zijl PC: Fiber tracking: principles and strategies - a technical review. NMR Biomed 15:468-480, 2002

33. Nimsky C, Ganslandt O, Hastreiter P, Wang R, Benner T, Sorensen AG, et al: Preoperative and intraoperative diffusion tensor imaging-based fiber tracking in glioma surgery. Neurosurgery 61 (1 Suppl): 178-186, 2007

34. Nimsky C, Ganslandt O, Merhof D, Sorensen AG, Fahlbusch $\mathrm{R}$ : Intraoperative visualization of the pyramidal tract by diffusion-tensor-imaging-based fiber tracking. Neuroimage 30:1219-1229, 2006

35. Picht T, Kombos T, Gramm HJ, Brock M, Suess O: Multimodal protocol for awake craniotomy in language cortex tumour surgery. Acta Neurochir (Wien) 148:127-138, 2006

36. Picht T, Krieg SM, Sollmann N, Rösler J, Niraula B, Neuvonen T, et al: A comparison of language mapping by preoperative navigated transcranial magnetic stimulation and 
direct cortical stimulation during awake surgery. Neurosurgery 72:808-819, 2013

37. Picht T, Mularski S, Kuehn B, Vajkoczy P, Kombos T, Suess $\mathrm{O}$ : Navigated transcranial magnetic stimulation for preoperative functional diagnostics in brain tumor surgery. Neurosurgery 65 (6 Suppl):93-99, 2009

38. Pierpaoli C, Jezzard P, Basser PJ, Barnett A, Di Chiro G: Diffusion tensor MR imaging of the human brain. Radiology 201:637-648, 1996

39. Pravatà E, Sestieri C, Colicchio G, Colosimo C, Romani GL, Caulo M: Functional connectivity MRI and post-operative language performance in temporal lobe epilepsy: initial experience. Neuroradiol J 27:158-162, 2014

40. Robles SG, Gatignol P, Lehéricy S, Duffau H: Long-term brain plasticity allowing a multistage surgical approach to World Health Organization Grade II gliomas in eloquent areas. J Neurosurg 109:615-624, 2008

41. Rogić M, Deletis V, Fernández-Conejero I: Inducing transient language disruptions by mapping of Broca's area with modified patterned repetitive transcranial magnetic stimulation protocol. J Neurosurg 120:1033-1041, 2014

42. Rösler J, Niraula B, Strack V, Zdunczyk A, Schilt S, Savolainen P, et al: Language mapping in healthy volunteers and brain tumor patients with a novel navigated TMS system: evidence of tumor-induced plasticity. Clin Neurophysiol 125:526-536, 2014

43. Rossini PM, Barker AT, Berardelli A, Caramia MD, Caruso G, Cracco RQ, et al: Non-invasive electrical and magnetic stimulation of the brain, spinal cord and roots: basic principles and procedures for routine clinical application. Report of an IFCN committee. Electroencephalogr Clin Neurophysiol 91:79-92, 1994

44. Ruohonen J, Karhu J: Navigated transcranial magnetic stimulation. Neurophysiol Clin 40:7-17, 2010

45. Salmelin R, Helenius P, Service E: Neurophysiology of fluent and impaired reading: a magnetoencephalographic approach. J Clin Neurophysiol 17:163-174, 2000

46. Sanai N, Berger MS: Intraoperative stimulation techniques for functional pathway preservation and glioma resection. Neurosurg Focus 28(2):E1, 2010

47. Sanai N, Mirzadeh Z, Berger MS: Functional outcome after language mapping for glioma resection. N Engl J Med 358:18-27, 2008

48. Saur D, Lange R, Baumgaertner A, Schraknepper V, Willmes $\mathrm{K}$, Rijntjes M, et al: Dynamics of language reorganization after stroke. Brain 129:1371-1384, 2006

49. Snodgrass JG, Vanderwart M: A standardized set of 260 pictures: norms for name agreement, image agreement, familiarity, and visual complexity. J Exp Psychol Hum Learn 6:174-215, 1980

50. Sollmann N, Giglhuber K, Tussis L, Meyer B, Ringel F, Krieg SM: nTMS-based DTI fiber tracking for language pathways correlates with language function and aphasia - A case report. Clin Neurol Neurosurg 136:25-28, 2015

51. Sollmann N, Hauck T, Hapfelmeier A, Meyer B, Ringel F, Krieg SM: Intra- and interobserver variability of language mapping by navigated transcranial magnetic brain stimulation. BMC Neurosci 14:150, 2013

52. Sollmann N, Ille S, Hauck T, Maurer S, Negwer C, Zimmer $\mathrm{C}$, et al: The impact of preoperative language mapping by repetitive navigated transcranial magnetic stimulation on the clinical course of brain tumor patients. BMC Cancer 15:261, 2015
53. Sollmann N, Picht T, Mäkelä JP, Meyer B, Ringel F, Krieg SM: Navigated transcranial magnetic stimulation for preoperative language mapping in a patient with a left frontoopercular glioblastoma. J Neurosurg 118:175-179, 2013

54. Sollmann N, Tanigawa N, Ringel F, Zimmer C, Meyer B, Krieg SM: Language and its right-hemispheric distribution in healthy brains: An investigation by repetitive transcranial magnetic stimulation. Neuroimage 102 (Pt 2):776-788, 2014

55. Szelényi A, Bello L, Duffau H, Fava E, Feigl GC, Galanda $\mathrm{M}$, et al: Intraoperative electrical stimulation in awake craniotomy: methodological aspects of current practice. Neurosurg Focus 28(2):E7, 2010

56. Tarapore PE, Findlay AM, Honma SM, Mizuiri D, Houde JF, Berger MS, et al: Language mapping with navigated repetitive TMS: proof of technique and validation. Neuroimage 82:260-272, 2013

57. Tuch DS, Reese TG, Wiegell MR, Wedeen VJ: Diffusion MRI of complex neural architecture. Neuron 40:885-895, 2003

58. van der Knaap LJ, van der Ham IJ: How does the corpus callosum mediate interhemispheric transfer? A review. Behav Brain Res 223:211-221, 2011

59. Wakana S, Jiang H, Nagae-Poetscher LM, van Zijl PC, Mori $\mathrm{S}$ : Fiber tract-based atlas of human white matter anatomy. Radiology 230:77-87, 2004

60. Wang L, Chen D, Yang X, Olson JJ, Gopinath K, Fan T, et al: Group independent component analysis and functional MRI examination of changes in language areas associated with brain tumors at different locations. PLoS One 8:e59657, 2013

61. Weiss C, Tursunova I, Neuschmelting V, Lockau H, Nettekoven C, Oros-Peusquens AM, et al: Improved nTMS- and DTI-derived CST tractography through anatomical ROI seeding on anterior pontine level compared to internal capsule. Neuroimage Clin 7:424-437, 2015

62. Wheat KL, Cornelissen PL, Sack AT, Schuhmann T, Goebel $\mathrm{R}$, Blomert L: Charting the functional relevance of Broca's area for visual word recognition and picture naming in Dutch using fMRI-guided TMS. Brain Lang 125:223-230, 2013

\section{Disclosures}

Drs. Krieg and Ringel are consultants for Brainlab AG. Dr. Krieg is also a consultant for Nexstim Oy.

\section{Author Contributions}

Conception and design: Krieg. Acquisition of data: Krieg, Sollmann, Negwer, Tussis, Hauck, Ille, Maurer, Giglhuber, Bauer. Analysis and interpretation of data: Krieg, Sollmann, Negwer, Tussis. Drafting the article: Krieg, Sollmann. Critically revising the article: Krieg, Sollmann. Reviewed submitted version of manuscript: Krieg, Hauck, Ille, Maurer, Giglhuber, Bauer, Ringel, Meyer. Approved the final version of the manuscript on behalf of all authors: Krieg. Statistical analysis: Krieg, Sollmann, Negwer. Administrative/technical/material support: Krieg, Ringel, Meyer. Study supervision: Krieg, Meyer.

\section{Correspondence}

Sandro M. Krieg, Department of Neurosurgery, Klinikum rechts der Isar, Technische Universität München, Ismaninger Str. 22, Munich 81675, Germany. email: sandro.krieg@tum.de. 